\title{
Methodology and Information Technology of Cyber-Physical-Socio Systems Integrated Modelling and Simulation
}

\author{
Boris Sokolov ${ }^{1,2}$ Mikhail B. Ignatyev ${ }^{2}$ Karim Benyamna $^{3} \quad$ Dmitri Ivanov $^{4} \quad$ Ekaterina Rostova $^{1}$ \\ ${ }^{1}$ St.Petersburg Institute for Informatics and Automation of the Russian Academy of Sciences (SPIIRAS), Russia \\ sokolov boriseinbox.ru, rostovae@mail.ru \\ ${ }^{2}$ St. Petersburg State University of Aerospace Instrumentation (SUAI), Russia \\ ignatmb@mail.ru \\ ${ }^{3}$ St. Petersburg National Research University of Information Technologies, Mechanics and Optics (ITMO Univ.), Russia \\ benyamna. karimegmail.com \\ ${ }^{4}$ Department of Business Administration, Berlin School of Economics and Law, Germany \\ dmitri.ivanov@mail.ru
}

\begin{abstract}
The main objects of our investigation are cyberphysical-socio space and systems (CPSS). The CPSS is the fusion of the physical space, the cyber space, and the social space. The problem of CPSS integrated modelling and simulation is an actual modern problem. The solution of this problem involves interdisciplinary research by specialists in mathematics, economics, biology, physics, and computer technologies. Therefore, the paper presents the results of research in the field of CPSS modelling and multi-agent simulation.
\end{abstract}

Keywords: interdisplinary research, cyber-physicalsocio space and systems, cybernetics, control and management, modelling and multi-agent simulation

\section{Introduction}

Today we come to the realization that a transformation from an industrial society to an informational society should be guided. Now we live in cyber-physical-socio space and systems (CPSS) (Lee, 2008). It is also called cyber-physical society (Zhuge, 2010). CPSS in contrast with cyber-physical systems (CPS) consist of not only cyberspace and physical space, but also human knowledge, mental capabilities, and sociocultural elements.

The technologies for control and management of transformation from an industrial society to an informational society need regulation and structuring at a macro and micro level. This inspires a renewed interest in the theoretical background of control and management problems. Unfortunately, logically relevant chain of fundamental notions: Cybernetics - Control Informational processes - Universal transformer of the information (computer, cybernetic machine) was split. An expansion of computer technologies caused an illusion of their ability to solve any problem. The imperfection of these technologies has already caused catastrophes that let American and European scientists proclaim establishing "Risk society" rather than "Informational" one.

Two main reasons stimulate importance of the new cybernetics in the modern world (Bir, 1963; Okhtilev et al, 2006). The first one deals with a problem of complexity which has various applications and aspects (structural complexity, complexity of functioning, complexity of decision making, etc.). The second reason is the lack of holistic (system) thinking in the IT industry. The problem of complexity control and management involve interdisciplinary research by specialists in mathematics, economics, sociology, biology, physics, and computer technologies. However, the founders of cybernetics viewed its laws as much more universal than they are really considered in today's social and business systems. Therefore, the paper presents the results of interdisciplinary research in the field of computer modeling and decision support systems in socialcybernetics objects. This field is called neocybernetics (von Foerster, 1987; Okhtilev, 2006).

\section{Interdisciplinary Branch of System Knowledge as the Basis for Cyber- Physical-Socio Systems Integrated Modelling and Simulation}

As a result of the continuing scientific and technical revolution which beginning is dated the middle of the $\mathrm{XX}$ century, there appeared a significant number of complex objects (nuclear power plants, space equipment, electronics, computers, etc.) research, the description, design and management of which presents essential difficulties and problems. CPSS are striking examples of complex systems. Figure 1 illustrates the evolution from the cyberspace and systems to the cyber-physical-social space and systems (Lee, 2008). In Figure 1 part (a) depicts two types of cyber space: the first one only allows users to read the information in the cyber space like the Web, and the other one allows users to read and write 
information in the cyber space like the Web 2.0. Both rely on humans to add information to the cyber space in order to share it with others. Part (b) in Figure 1 depicts the extension of the cyber space to the physical space through various sensors. Any significant information in the physical space can be automatically sensed, stored and transmitted through the cyber space. Web of things can be considered as a kind of cyber-physical space. Part (c) in Figure 1 depicts an important extension of part (b): user behaviors can be sensed and feedbacked to the cyber space for analyzing the patterns of behaviors, and humans can remotely control the actuators to behave in the physical space through the cyber space. This enables the cyber space to adapt its services according to the feedback since behavior change may indicate some psychological change (Zhuge, 2010). Part (d) in Figure 1 depicts a simple cyber-physical-social space. Not only individual's behaviors, but also social interactions can be feedback into the cyberspace for further processing. Users are considered with their social characteristics and relations rather than as isolated individuals. Sensors are limited in their ability to collect all information in the physical space, so users still need to directly collect the significant information in the physical space and then put it into the cyberspace after analysis (including experiment). Users can also manipulate physical objects in the physical space, which can also be feedbacked into the cyber space to reflect the real-time situation (Zhuge, 2011). Users' status, interests and knowledge evolve with social interaction and operations in the cyber space.

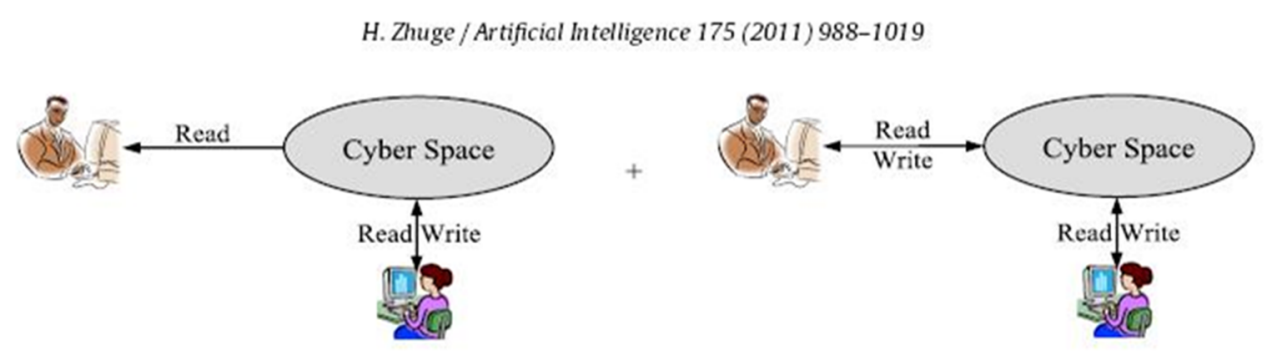

(a) The cyber space.

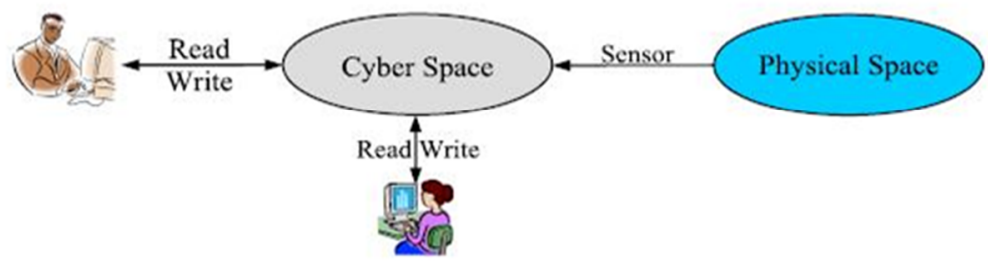

(b) The cyber-physical space.

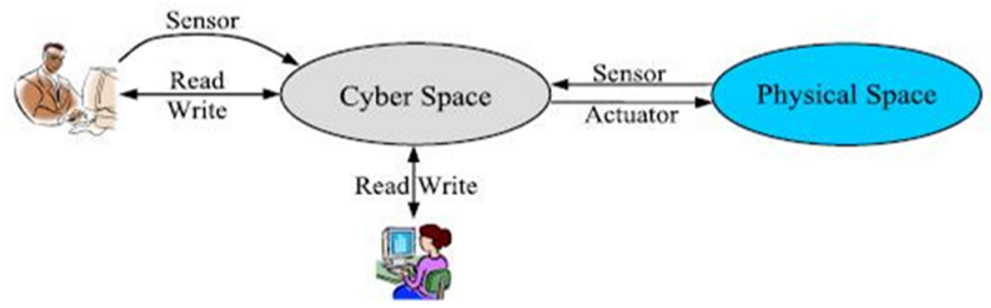

(c) The cyber-physical-human space.

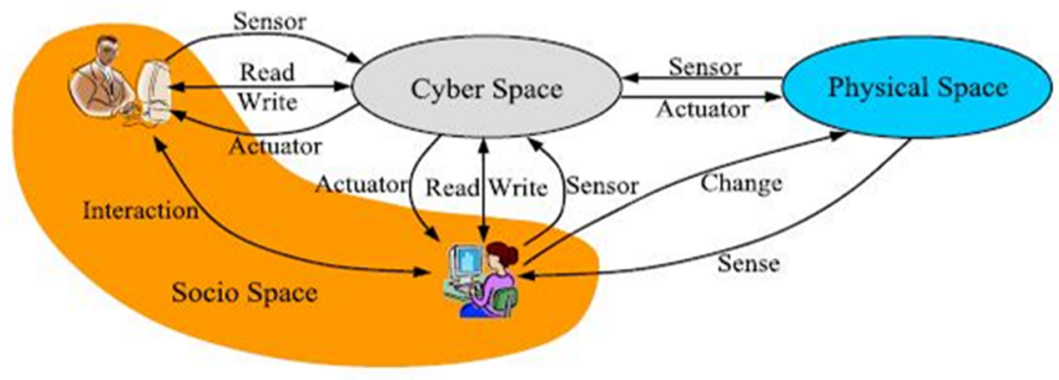

(d) The cyber-physical-socio space.

Figure 1. The emerging cyber-physical-socio space. 
So today, we come to the realization of a transformation from an industrial society which is based on cyber-physical space to an informational society which is interrelated with cyber-physical-socio space and systems. The technologies for control of this transformation need regulation and structuring at a macro and micro level. This inspires a renewed interest in the theoretical and practical background of complexity control and management problems. There exist different aspects of CPSS. For example, CPSS are characterized by following properties: high complexity and dimensionality with such features as redundancy, multifunctionality, distributed elements, unification, uniformity of main elements, subsystems and interrelations; structure dynamics; nonlinear uncertain behavior; hierarchical network structure; nonequilibrium; uncertainty of observer selection and interaction with him; dynamic rules and regulations; counteracting and amplifying relationships with self-excitation; possible chaotic behavior; no element has enough information about the whole system; selective sensitivity to input actions (dynamic robustness and adaptation); the response time is greater than the time between input actions, and it is greater than the time of supervention; the real object of control cannot have a complete and reliable description (in accordance with Bremerman's limit and Godel's theorem) (Okhtilev et al, 2006; Ignatyev, 2008). The specified objective circumstances which are connected with CPSS have resulted in need at the beginning of the XXI century of formation in a wide range of experts of system outlook and the relevant system branch of scientific knowledge. It has been shown in the works (Lee, 2008; Ignatyev, 2008) that speaking about the interdisciplinary branch of the system's knowledge, it is expedient to select in it two big sections (block) - the block of fundamental system info-cybernetic knowledge and the block of applied system info-cybernetic knowledge. In the first of the listed blocks, the defining role is played by three scientific directions - the general theory of systems, cybernetics and informatics.

As for the general theory of systems (systemology), this scientific direction sets the task to construct the general scientific basis for systems of any nature. The central concepts of the general theory of systems are the concepts of an open system, i.e. the system interacting with the environment surrounding it, both complex and big. The mathematical basis for the general theory of systems can reasonably enough be considered as a certain interpretation of the basis for mathematics, mainly theories of the relations (the concept of the relation is fundamental both in mathematics, and in system researches), theories of mathematical structures and the theory of categories and functors. However, except for a number of recognized general provisions in general for the present, there is no uniform understanding of in what way this theory has to be applied.

As regards informatics, this scientific direction is connected with the development of methods and means of collecting, storage, transfer, representation, processing and information security. Speaking about the processes of interaction of cybernetics with informatics it should be noted, first, that historically the last one considerably developed into a subsoil of traditional cybernetics, actually on uniform technical base - computer facilities and means of communication and data transmission, and, secondly, cybernetics, being a science about the general laws and regularities of management and communication, has objectively been forced in recent years to deal with the second round of rapprochement of cybernetics and informatics. There is an active terminological and substantial interpenetration of these scientific directions, issues of use of information for the benefit of management. Therefore, the methods, technologies and means developed into an informatics that actively take root into cybernetics within such new scientific directions as: information management, different types of intellectual management (situational, neuromanagement, the management based on knowledge, on the basis of evolutionary algorithms, multi-agent management and etc.). These types of intellectual management are based, in turn, on the appropriate intellectual information technologies (IIT) focused on symbolic information processing.

Cybernetics is the general theory of management. Initially with the founder of cybernetics N. Winer in 1948 in his book "Cybernetics or Management and Communication in an Animal and the Car", it was emphasized that this science is about management, communication and processing of information in systems of any nature (Wiener, 1950). At the same time, the main goal of the research conducted within the specified science consisted in identification and establishment of the most general laws of functioning to which submit as the operated objects, and the corresponding managing directors of a subsystem without regard to their nature. The classical cybernetics has reduced all earlier existing views of management processes into uniform systems and has proved its completeness and generality. In other words, it has shown in detail the raised power of system approach to the solution of complex problems (Ignatyev, 2008). The most developed direction in cybernetics was the theory of management of dynamic technical systems within which numerous outstanding fundamental and applied scientific results have been received by multiple experts (Heikki, 2006; Okhtilev et al, 2006; Ignatyev, 2008; Zhuge, 2010, 2011).

In turn, cybernetic terminology gets into informatics and computer facilities. Today, in particular, concepts and, respectively, strategies of adaptive and proactive computer systems, adaptive management and the adaptive enterprise are very popular in the IT industry. Those strategies are intensively developed by the companies IBM, Intel Research, Hewlett-Packard, Microsoft, Sun, etc. (Okhtilev et al, 2006). At the same time, the material basis for the realization of technologies of the operated self-organization is created. In the modern business systems (BS) only those organizations obtain success in which development of IT architecture is focused on the Web services and technologies allowing to effectively decentralize traditional systems of decision-making, turning them into self-regulating subsystems. Interaction of cybernetics (neocybernetics) and informatics with the 
general theory of systems is carried out in several directions. The first of these directions is directly connected with the generalized description of objects and subjects of management on the basis of the new formalistic approaches developed in a modern systemology to which it is possible to refer, for example, structural and mathematical and category-functor's approaches (Okhtilev et al, 2006). In this regard, it is also possible to note interesting scientific results which have been received in a qualimetry of models and poly-model complexes and can be used in informatics and cybernetics. The methods and algorithms of decomposition (composition), aggregation (disaggregations), and coordination developed in the general theory of systems in relation to objects of any nature are widely used in cybernetics and informatics also in the solution of the problems of collecting, storage, transfer, representation, processing, information security, and also management of complex objects. On the other hand, it has been shown in the works (Wiener, 1950; Foerster, 1987; Okhtilev, 2006) that the approaches developed in the classical theory of management of technical objects and also in modern informatics can be applied successfully to the organization of processes of management of quality of models and poly-model complexes, and also at their structural and parametric adaptation.

\section{Methodological and Technical Basis for CPSS Integrated Modelling and Simulation}

During our investigation, we describe the main classes of CPSS integrated modeling tasks. For these aims, we use structure-dynamics control (SDC) theory. The methodological basis of this theory includes the methodologies of generalized system analysis and the modern optimal control theory for CTS with reconfigurable structures. The dynamic interpretation of SDC processes lets apply the results, previously received in the theory of dynamic system's stability, ability, failure tolerance, effectiveness and sensitivity, for CPSS analysis problems. The existence of various alternative descriptions for CPSS elements and control subsystems gives an opportunity of adaptive model selection (synthesis) for program control in changing environment. Therefore, we considered two general actual problems of the CPSS structure-dynamics investigation. Those are the problem of selection of optimal CPSS structure-dynamics control programs at different states of the environment and the problem of parametric and structural adaptation of models describing CPSS structure-dynamics control. In this case the adaptive control should include the following main phases: parametric and structural adaptation of SDC models and algorithms to previous and current states of objects-in-service (SO), of control subsystems (CS), and of the environment; integrated scheduling of CPSS operation (construction of SDC programs); simulation of CPSS operation, according to the schedules, for different variants of control decisions in real situations; structural and parametric adaptation of the schedule, control inputs, models, algorithms, and CPSS programs to possible (predicted by simulation) states of SO, CS, and the environment.

During our investigations, the main phases and steps of a program construction procedure for optimal structure-dynamics control in CPSS were proposed (Okhtilev et al, 2006). At the first phase forming (generation) of allowable multi-structural macro-states is being performed. In other words, a structure-functional synthesis of a new CPSS make-up should be performed in accordance with an actual or forecasted situation. Here the first-phase problems come to CPSS structurefunctional synthesis. In the second phase, a single multistructural macro-state is being selected, and adaptive plans (programs) of the CPSS transition to the selected macro-state are being constructed. These plans should specify transition programs, as well as programs of stable CPSS operation in intermediate multi-structural macrostates. The second phase of program construction is aimed at a solution of multi-level multi-stage optimization problems.

One of the main opportunities of the proposed method of CPSS SDC program construction is that besides the vector of program control, we receive a preferable multistructural macro-state of the CPSS at the end point. This is the state of CPSS reliable operation in the current (forecasted) situation. The combined methods and algorithms of optimal program construction for structuredynamics control in centralized and non-centralized modes of CPSS operation were developed (Okhtilev et al, 2006). Classification and analysis of perturbation factors having an influence upon the operation of CPSS were performed. Variants of perturbation-factors descriptions were considered in CPSS SDC models. In our opinion, an integrated simulation of uncertainty factors with all adequate models and forms of description should be used during the investigation of CPSS SDC. Moreover, the abilities of CPSS management should be estimated in both normal mode of operation and emergency situations. It is important to estimate the destruction "abilities" of perturbation impacts. In this case, the investigation of CPSS functioning should include the following phases: determining of scenarios for CPSS environment, particularly determining of extreme situations and impacts that can have catastrophic results; analysis of CPSS operation in a normal mode on the basis of a priori probability information (if any), simulation, and processing of expert information through the theory of subjective probabilities and theory of fuzzy sets; repetition of item $b$ for the main extreme situations and estimation of guaranteed results of CPSS operation in these situations; computing of general (integral) efficiency measures of CPSS structure-dynamics control. Algorithms of parametric and structural adaptation of CPSS SDC models were proposed (Okhtilev et al, 2006). The algorithms will be based on the methods of fuzzy clusterization, on the methods of hierarchy analysis, on biological adaptation mechanisms, and on the methods of a joint use of analytical and simulation models. We illustrate our methodology by two examples. 


\section{Example of Lingvo-Combinatorial Modelling}

We often use a natural language to describe systems. We propose to transfer this natural language description to mathematical equations.

For example, we have a sentence:

$$
\text { WORD } 1+W O R D 2+W O R D 3
$$

Here we assign words and only imply the meaning of words, the meaning (sense) is only implied but not designated.

We propose to assign meaning in the following form:

$$
\begin{gathered}
(\text { WORD1 }) *(S E N S E 1)+(\text { WORD2 }) * \\
(S E N S E 2)+(\text { WORD3) * (SENSE } 3)=0
\end{gathered}
$$

This equation (2) can be represented in the following form:

$$
A 1 * E 1+A 2 * E 2+A 3 * E 3=0
$$

Where Ai, i = 1, 2, 3, will denote words from English Appearance and Ei will denote senses from English Essence. The equations (2) and (3) are the model of the sentence (1). When we have a mathematical equation in the form $F(x 1, x 2, x 3)=0$, we can turn such a form by means of differentiation where the partial derivatives are the appearances and the derivatives with respect to time are the essences. This model is an algebraic ring and we can resolve this equation with respect to the appearances $\mathrm{Ai}$ or the essences Ei (Ignatyev, 2008):

$$
\begin{aligned}
& A 1=U 1^{*} E 2-U 2^{*} E 3 \\
& A 2=-U 1^{*} E 1+U 3^{*} E 3 \\
& A 3=-U 2^{*} E 1-U 3^{*} E 2
\end{aligned}
$$

or

$$
\begin{aligned}
& A 1=U 1^{*} A 2-U 2^{*} A 3 \\
& A 2=-U 1^{*} A 1+U 3^{*} A 3 \\
& A 3=-U 2^{*} A 1-U 3^{*} A 2
\end{aligned}
$$

\begin{tabular}{|c|c|c|c|c|c|c|c|c|}
\hline $\mathbf{n} / \mathbf{m}$ & 1 & 2 & 3 & 4 & 5 & 6 & 7 & 8 \\
\hline 2 & 1 & & & & & & & \\
\hline 3 & 3 & 1 & & & & & & \\
\hline 4 & 6 & 4 & 1 & & & & & \\
\hline 5 & 10 & 10 & 5 & 1 & & & & \\
\hline 6 & 15 & 20 & 15 & 6 & 1 & & & \\
\hline 7 & 21 & 35 & 35 & 21 & 7 & 1 & & \\
\hline 8 & 28 & 56 & 70 & 56 & 28 & 8 & 1 & \\
\hline 9 & 36 & 84 & 126 & 126 & 84 & 36 & 9 & 1 \\
\hline
\end{tabular}

Where U1, U2, U3 are arbitrary coefficients, can be used for solution of different tasks on the initial manifold (2) or (3). In general, if we have n variables in our system and $\mathrm{m}$ manifolds, constraints, then the number of arbitrary coefficients $\mathrm{S}$ will be defined as the number of combinations from $\mathrm{n}$ to $\mathrm{m}+1$ (Ignatyev, 2008), as shown in Table 1.
Table 1. Number of combinations from $\mathrm{N}$ to $\mathrm{M}+1$.

$$
S=C_{n}^{m+1} n>m
$$

The formula (6) is the basic law of cybernetics, informatics and synergetics for complex systems (Ignatyev, 2008). The number of arbitrary coefficients is the measure of uncertainty. Usually, when solving mathematical systems, the number of variables is equal to the number of equations. In practice, we frequently do not know how many constraints, there are on our variables. Combinatorial simulation makes it possible to simulate and study the systems with uncertainty on the basis of incomplete information. The problem of simulation of condition, guaranteeing the existence of maximum adaptability, is considered.

It is supposed that the behavior of a system with $n$ variables is given with an accuracy of $\mathrm{m}$ intersecting manifolds, $\mathrm{n}>\mathrm{m}$. If the system is considered as a multidimensional generator where at least a part of the variables interacts with environment variables, and if the objective of the system is to decrease the functional of discoordination between them ( $\square 1 \ldots \square \mathrm{k})$, the system control unit has two instruments of impact, $a$ and $b$, upon the system. First, this is the tuning - the changing of uncertain coefficients in the structure of the differential equations of the system, taking into account that the greater number of these coefficients implies a more accurate system response to a changing environment. Second, this is the learning - the imposing new restrictions on the system behavior. The number of arbitrary coefficients, in the structure of equivalent equations, changes in the process of learning, of consecutive imposing new and new restrictions on the system behavior. In the systems with more than six variables the number of arbitrary coefficients increases first, and then, passing through the maximum begins to decrease. This phenomenon makes it possible to explain the processes of system growth, complication and death. The existence of maximum adaptability phenomenon is observed in numerous biological, economical and physical-engineering systems. It is important that we describe a system with a full sum of combinations and 
have all the variants of decisions. The linguocombinatorial simulation is a useful heuristic approach for investigation of complex, poorly formalized systems. Natural language is the main intellectual product of mankind; the structure of natural language reflects the structure of natural intellect of mankind and its certain representatives on the level of consciousness and unconsciousness. Linguo-combinatorial simulation is the calculation, which allows to extract the senses from texts. L. Wittgenstein wanted to have the calculation of senses. In our calculation we have the three groups of variables: the first group - the words of natural language $\mathrm{Ai}$, the second group - the essences $\mathrm{Ei}$, which can be the internal language of brain (Ignatyev, 2008); we can have the different natural languages, but we have only one internal language of brain; this hypothesis opens a new way for experimental investigation; the third group of variables - the arbitrary coefficients, structural uncertainty in our model, which we can use for adaptation in translation processes and etc.

\section{Example of Transport Networks Simulation}

As an example of the integrated modeling and simulation based on the CPSS, we developed a simulation program (Figure 2) and an experimental stand (Figure 3) with a transport network (for example, railroad) and some production and warehouse facilities are currently under development. The railroad is provided with multiple sensors, for example, the RFID, which provides information about the position and the speed of bypassing locomotives.

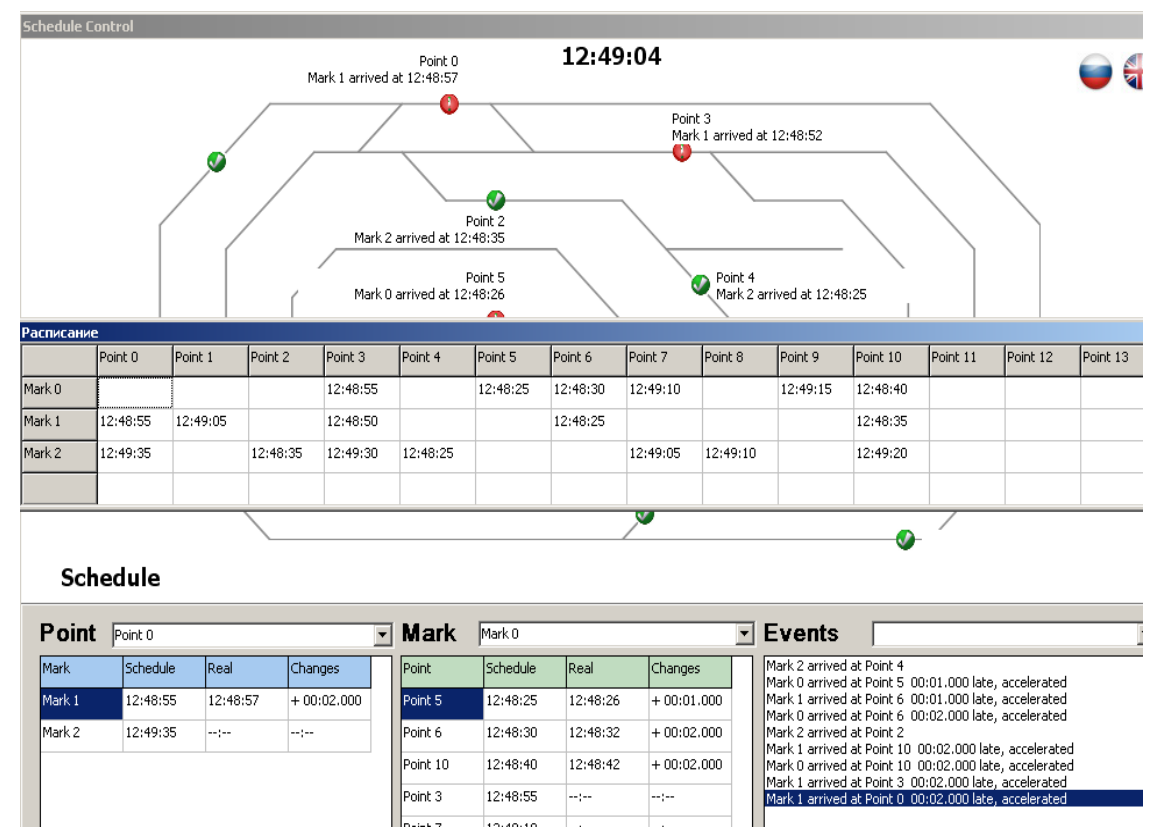

Figure 2. Screenshot from the transport network simulation program.

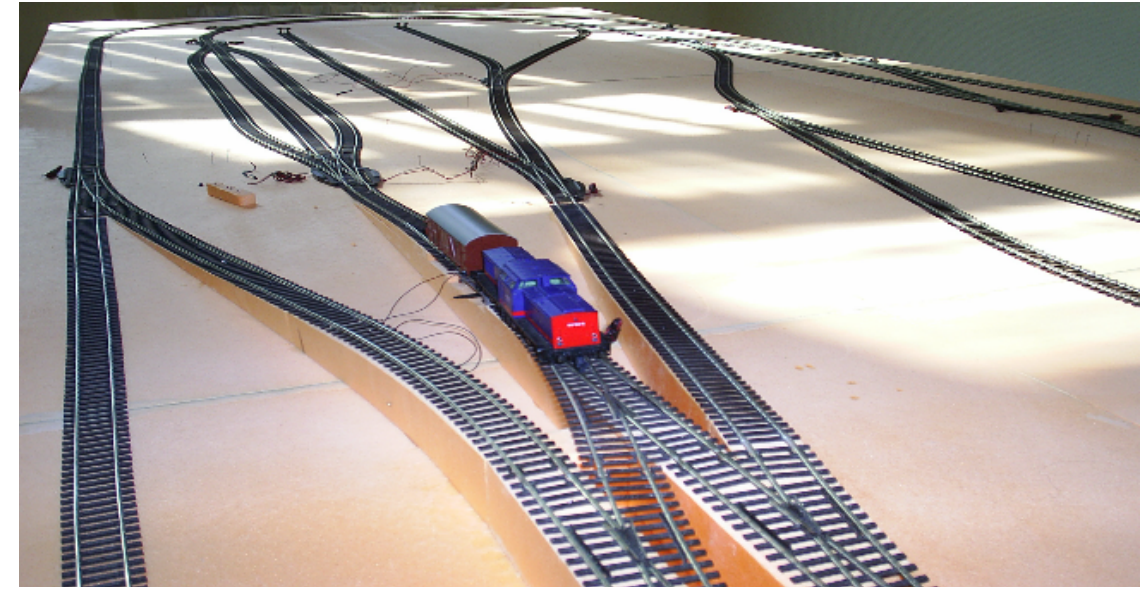

Figure 3. The experimental stand. 
We note that the RFID experimental environment is not intended (at least, in its current version) for a full implementation of the developed models. It is much simpler than the modeling framework and serves to gather experimental data for the modeling complex. The modeling complex itself is implemented in a special software environment, which contains a simulation and optimization engine of CS planning and execution control, a Web platform, an ERP system, and APS system, and a SCEM system. The kernel of the computational framework is the decision modeling component, that is, the simulation and optimization engine. The schedule optimization is based on an optimal control algorithm that is launched by a heuristic solution, the so-called first approach. The seeking for the optimality and the CS scheduling level is enhanced by simultaneous optimizing and balancing interrelated CS functional, organizational and information structures. The schedules can be analyzed with regard to performance indicators and different execution scenarios with different perturbations. Subsequently, parameters of the CS structures and the environment can be tuned if the decision-maker is not satisfied with the values of performance indicators. In analyzing the impact of the scale and location of the adaptation steps on the CS performance, it becomes possible to justify methodically the requirements for the RFID functionalities, the stages of a CS for the RFID element locations, and the processing information from RFID. In particular, possible discrepancies between actual needs for a wireless solution of CS control problems and the total costs of ownership regarding RFID can be analyzed. In addition, processing information from RFID can be subordinated to different management and operation decision-making levels (according to the developed multi-loop adaptation framework). Pilot RFID devices with reconfigurable functional structure are developed (Kurve et al, 2013). In order to simulate the RFID-based transport network, we have created a prototype of a simulation model that reproduces a real railway network. To do this, we could use different approaches, but the most suitable are the multi-agent system (MAS) modelling (Niazi et al, 2011; Kurve et al, 2013). To realize the simulation model, we could use different tools made for modeling a MAS (Okhtilev et al, 2006; Lee, 2008; Niazi et al, 2011; Kurve et al, 2013) but most of them have a restrictive or a paid licensing policy. So we managed to develop from scratch a prototype of a simulation environment based on the $\mathrm{C}++$ programming language and the MAS approaches. The first step in creating a simulation model is to define the time framework (Niazi et al, 2011) implemented in the form of a main event loop. In the loop, we have two functions. The first may or may not create a random disturbance in the locomotive speed and the maximum speed. The second function simulates the agent behavior. This system enables us to simulate a basic railroad network and test the RFID infrastructure on a realistic model. Of course, as any system, this one has its advantages and disadvantages. As regards advantages, they include: the technologies used to create this model $(\mathrm{C}++$ language $)$, it provides us with a great flexibility in terms of functionality, allowing for modification and implementation of any kind of logic we want; agent-based modeling is a powerful method allowing a large number of enhancements in the behavior of the system. Additionally, it enables us to define a logic of each individual locomotive, which is close to how decisions are made in a real system; define the system behavior by an independent entity allows greater scalability, as the complexity of the system is linear to the number of entities.

\section{Conclusions}

Dynamic multiple-model descriptions of CPSS functioning at different stages of their lifecycle are proposed in the paper. Different types of models (analytical-simulation, logical algebraic, logicallinguistic models) were proposed for description and study of the main attributes of the CPSS (Yusupov et al, 2011; Geida et al, 2015; Yusupov, 2009). Joint use of diverse models in the framework of poly-model systems, allows one to improve the flexibility and adaptability of IDSS, as well as to compensate the drawbacks of one class of models by the advantages of the other (Okhtilev et al, 2006; Ignatyev, 2008).

\section{Acknowledgements}

The research described in this paper is partially supported by the Russian Foundation for Basic Research (grants 1507-08391, 15-08-08459, 16-07-00779, 16-08-00510, 1608-01277, 16-29-09482-ofi-i, 17-08-00797, 17-0600108, 17-01-00139, 17-20-01214, 17-29-07073-ofi-i), grant 074-U01 (ITMO University), state order of the Ministry of Education and Science of the Russian Federation №2.3135.2017/4.6, state research 00732014-0009, 0073-2015-0007, International project ERASMUS +, Capacity building in higher education, № 73751-EPP-1-2016-1-DE-EPPKA2-CBHE-JP, Innovative teaching and learning strategies in open modelling and simulation environment for studentcentered engineering education.

\section{References}

S. Bir. Cybernetics and production control. Fizmatlit. 1963. H. von Foerster. Cybernetics. Encyclopedia of Artificial Intelligence. John Wiley and Sons. 1987.

A.S. Geida, I.V. Lysenko, and R.M Yusupov. Main Concepts and Principles for Information Technologies Operational Properties Research. SPIIRAS Proceedings, 42:5-36, 2015.

H. Heikki. Neocybernetics in Biological Systems. Helsinki University of Technology, Control Engineering Laboratory. Report 151, p. 273, August 2006.

M.B. Ignatyev. Semantics and selforganization in nanoscale physics. International Journal of Computing Anticipatory Systems, 22:17-23, 2008.

A. Kurve, K. Kotobi, and G. Kesidis. An agent-based framework for performance modeling of an optimistic parallel discrete event simulator. Complex Adaptive Systems Modeling, 1(1):12, 2013. doi: 10.1186/21943206-1-12

E.A. Lee. Cyber physical systems: Design challenges. 2008 11th IEEE International Symposium on Object and 
Component-Oriented Real-Time Distributed Computing (ISORC), 11:363-369, 2008.

doi: 10.1109/ISORC.2008.25

M. Niazi, A. Hussain. Agent-based computing from multiagent systems to agent-based models: a visual survey. Scientometrics, 89(2):479-499, 2011.

doi: $10.1007 / \mathrm{s} 11192-011-0468-9$

M.Yu. Okhtilev, B.V. Sokolov, and R.M. Yusupov. Intellectual technologies for monitoring and control of structural dynamics of complex technical plants. Nauka Publishers. 2006.

N. Wiener. The Human Use of Human Beings: Cybernetics and Society. Da Capo Press. 1950.

R.M. Yusupov, B.V. Sokolov, A.I. Ptushkin, A.V. Ikonnikova, S.A. Potryasaev, and E.G.Tsivirko. Research problems analysis of artificial objects lifecycle management. SPIIRAS Proceedings, 16:37-109, 2011.

R.M. Yusupov. About the impact of information and communication technologies on the national security assurance in the environment of the information society forming. SPIIRAS Proceedings, 8:21-33, 2009.

H. Zhuge. Cyber physical society. Proceedings of the 6th International Conference on Semantics, Knowledge and Grids, 1:1-8, 2010.

H. Zhuge. Semantic linking through spaces for cyber-physicalsocio intelligence: a methodology. Artificial Intelligence, 175:988-1019, 2011. doi: 10.1016/j.artint.2010.09.009 